There were not intraoperative complications Orthotopic ileas reconstruction is a complex technique, but it allows a more anatomical reconstruction, avoids the creation of stoma with less impact on the quality of life. It requires integrity of the sphincter and bladder neck and should be assessed and discussed thoroughly with the patient against other possibilities. Conclusion* It is essential to balance the radicality of the best surgical result with the least posible impact on quality of life

Our next goal shoud be to obtain the best oncological results and survival with the minimum complications and consequences for our patients.

\section{CARTER DOUBLE-BARRELED WET COLOSTOMY AS A SURGICAL RECONSTRUCTION METHOD AFTER PELVIC EXENTERATION}

T Echim* Z Z Novák. National Intitute of Oncology, Budapest, Hungary

\subsection{6/ijgc-2021-ESGO.586}

Introduction/Background* Carter double-barreled wet colostomy (DBWC) is an innovative technique frequently used for pelvic exenteration. The main advantage of this surgical approach is that it keeps the fecal and urine streams separate, thus avoiding fecal reflux and subsequently reducing the risk of ascending pyelonephritis.

Methodology Our aim was to investigate the impact BDWC on quality of life (QOL) after total pelvic exenteration. In our prospective study, self-reported QOL was assessed with the EuroQol 5 Dimensions (EQ5D) QOL questionnaire.

Result(s)* In 2019, two patients underwent total pelvic exenteration involving BDWC in our Institution. Both patients reported an improved QOL after surgery.

Conclusion* Altogether, our findings support the use of the relatively simple and safe DBWC technique in pelvic tumors. Nevertheless, further large-scale studies are warranted to investigate the impact of DBWC on short- and long-term postoperative outcomes, QOL and survival.

\section{Translational research biomarkers}

\section{THE PROGNOSTIC SIGNIFICANCE OF EUKARYOTIC TRANSLATION INITIATION FACTORS (EIFS) IN OVARIAN CANCER}

\begin{abstract}
${ }^{1,2} \mathrm{M}$ Sobočan*, ${ }^{3} \mathrm{~S}$ Sprung, ${ }^{3} \mathrm{C}$ Schatz, ${ }^{1,2} \mathrm{~J} \mathrm{Knez},{ }^{2,4} \mathrm{R}$ Kavalar, ${ }^{2,4} \mathrm{I}$ Takač, ${ }^{3,5} \mathrm{~J}$ Haybaeck. ${ }^{1}$ University Medical Centre Maribor, Division for Gynaecology and Perinatology, Maribor, Slovenia; ${ }^{2}$ University of Maribor, Faculty of Medicine, Maribor, Slovenia; ${ }^{3}$ Medical University of Innsbruck, Institute of Pathology, Neuropathology and Molecular Pathology, Innsbruck, Austria; ${ }^{4}$ University Medical Centre Maribor, Department of Pathology, Maribor, Slovenia; ${ }^{5}$ Medical University Graz, Diagnostic and Research Center for Molecular BioMedicine, Department of Neuropathology, Diagnostic and Research Institute of Pathology, Graz, Austria
\end{abstract}

\subsection{6/ijgc-2021-ESG0.587}

Introduction/Background* Ovarian cancer represents the most lethal gynaecological cancer. Although treatment options for patients with ovarian cancer have expanded, many patients suffer from disease relapse early after primary treatment. Different targeted therapies based on signalling pathways in ovarian cancer have yielded limited clinical success warranting the evaluation of further biological targets to improve therapy precision. A potential target is the machinery of protein synthesis, facilitated by eukaryotic initiation factors (eIFs). However, little is known about the role of eIFs in ovarian cancer. The aim of this study was to evaluate the role of different eIFs and their correlation to clinical outcome in ovarian cancer patients.

Methodology We performed immunohistochemical staining for the 6 eIF subunits (eIF1A1, eIF2alpha, eIF2G, eIF5A, eIF5B and eIF6) from samples of women diagnosed with epithelial ovarian cancer (EOC) at the University Medical Centre Maribor, Slovenia between January 2009 and December 2014. For all samples, a composite score of density and intensity of expression was calculated. Expression data was assessed in correlation to recurrence free survival (RFS) and overall patient survival (OS). The statistical analysis was performed using the Spearman rank correlation.

Result(s)* The cohort consisted of 75 women with EOC with a mean age of 61.2 years (SD 11.15). Disease specific death occurred in $74 \%$ of women $(n=56)$ and disease recurred in $61 \% \quad(n=47)$ women. The eIF subunit eIF5A $\quad\left(r_{s}=-.234\right.$, $\mathrm{p}<0.043$ ) was found to be correlated with overall survival and recurrence free survival $\left(\mathrm{r}_{\mathrm{s}}=-.247, \mathrm{p}>0.033\right)$ in patients with EOC. An overexpression of eIF5A was significantly correlated with RFS $(U=496.5, p>0.017)$ and OS $(U=398.0$, $\mathrm{p}>0.006$ ).

Conclusion* Further evaluation of the initiation translation cascade in ovarian cancer and specifically the impact the expression of eIF5A has on EOC may be warranted. eIF5A may serve as a prognostic marker in EOC.

\section{ANALYSIS OF CONSECUTIVE HIGH-GRADE SEROUS OVARIAN CANCER PATIENTS ALLOWS EFFICIENT CATALOGING OF BRCA1/2 MUTATIONS IN YET UNSTUDIED ETHNIC GROUPS}

${ }^{1} \mathrm{~A}$ Sokolenko, ${ }^{2} \mathrm{~L}$ Sultanova, ${ }^{2} \mathrm{M}$ Tovgereyeva, ${ }^{3} \mathrm{E}$ Suleymanov, ${ }^{1} \mathrm{I}$ Stepanov, ${ }^{1} \mathrm{E}$ Vasilyeva, ${ }^{1} \mathrm{E}$ Bakaeva, ${ }^{1} \mathrm{E}$ Imyanitov*. ${ }^{1}$ N.N. Petrov Institute of Oncology, St.-Petersburg, Russian Federation; ${ }^{2}$ Chechen Republican Cancer Center, Grozny, Russian Federation; ${ }^{3}$ Ministry of Health of Chechen Republic, Grozny, Russian Federation

\subsection{6/ijgc-2021-ESG0.588}

Introduction/Background* Approximately 15-30\% high-grade serous ovarian carcinomas (HGSOCs) are caused by BRCA1/2 germ-line mutations. BRCA1/2 testing of consecutive HGSOC cases is logistically less complicated than the search for women with other clinical signs of BRCA1/2 syndrome (e.g., family history, young age or emergence of multiple cancers). We reasoned that this would be the most straightforward approach to identify ethnicity-specific mutations and investigated patients of Chechen origin. Chechens are a Northeast Caucasian ethnic group consisting of approximately 2 million people. This community is characterized by carefully preserved national and religious traditions, with a relatively low rate of interethnic marriage, and, consequently, high probability of persistence of founder alleles.

Methodology Coding sequences of BRCA1, BRCA2, ATM, TP53 and PALB2 genes were analyzed by next generation sequencing.

Result(s)* We initially included in the study 67 consecutive Chechen patients with HGSOC. Pathogenic BRCA1/2 alleles were detected in 12/67 (18\%) HGSOC cases; all 8 women with BRCA1 mutation carried the same pathogenic variant (c.3627_3628delAG), while some genetic diversity was 